\title{
Zertifizierte multidisziplinäre Brustzentren: Ein Implementierungsprojekt der Deutschen Krebsgesellschaft und der Deutschen Gesellschaft für Senologie in Partnerschaft mit der Deutschen Gesellschaft für Gynäkologie und Geburtshilfe
}

\section{Wallwiener, S. Y. Brucker}

Um die Qualität von Strukturen, Prozessen und Ergebnissen bei der Diagnostik und Behandlung des Mammakarzinoms zu sichern, hat die Deutsche Gesellschaft für Senologie und die Deutsche Krebsgesellschaft mit Hilfe des IFG ein multimodales System zur Zertifizierung von Brustzentren entwickelt und implementiert.

Die ersten Kliniken wurden nach diesem Schema zertifiziert. Inzwischen sind es bundesweit über 250Zentren. Das Internet-Portal Wikipedia hat den zertizifierten Brustzentren eine ausführliche eigene Seite gewidmet - ein Zeichen dafür, dass zertifizierte Brustzentren heute, nach erstaunlich kurzer Einführungszeit, aus der Versorgungswirklichkeit in Deutschland nicht mehr wegzudenken sind.

\section{Einleitung \\ $\nabla$}

Das Mammakarzinom ist nach wie vor die häufigste maligne Tumorerkrankung der Frau. Nach Schätzungen des Robert Koch-Instituts erkranken in der Bundesrepublik jährlich etwa 47500 Frauen neu an Brustkrebs [11], andere Quellen gehen von etwa 55000 Neuerkrankungen pro Jahr aus [4-6]. Das mittlere Brustkrebs-Lebenszeitrisiko beträgt in Deutschland 9,2\%; es erkrankt somit durchschnittlich jede 11. Frau im Lauf ihres Lebens an Brustkrebs [11].

In einer Mehrschrittstudienkonzeption sollte iterativ die Voraussetzungen geschaffen werden, die Schnittstellen der Versorgungskette des Mammakarzinoms zu definieren, standardisierte Diagnose- und Therapieverfahren im Sinne von Leitlinien vorzugeben, die Bedeutung der Multidisziplinarität zu analysieren und auf der Basis der evidenzbasierten Medizin eine Datenlage zu schaffen, die Standard für die Zentralisierung der Therapie von Mammakarzinompatientinnen zu ermöglichen und die Behandlungsqualität zu überprüfen und damit die Zentren zertifizieren zu können.

Im Einzelnen sollen zum Erreichen dieser Zielvorgaben die folgenden vier Kernfragen beantwortet werden:

- Welche Rationale ergibt sich für die Zentralisierung der Diagnostik und Therapie onkologischer Erkrankungen?

- Lassen sich Qualitätsindikatoren definieren und darauf ein Benchmarking aufbauen zur Erfassung der Versorgungsqualität (Proof of Principle)?

- Kann durch die Implementierung eines Qualitätsmanagementsystems zur Optimierung der Struktur-, Prozess- und Ergebnisqualität die Leitlinientreue nachgewiesen werden und ein Netzwerk qualitätsgesicherter und damit zertifizierbarer interdisziplinärer Brustzentren auf nationaler Ebene geschaffen werden (Proof of Concept)?

- Sind entsprechende Analysen auch für einzelne Querschnittsfächer wie die Radioonkologie möglich?

\section{Rationale für eine Zentrumsbildung}

$\nabla$

Diesbezüglich haben die letzten ca. 5Jahre gezeigt, dass anhand der Daten aus diesen Zentren vor allem die folgenden 3 Kernaussagen getroffen werden können, die zu einer Verbesserung der Qualität führen:

- Verbesserung des Outcome mit steigender Zahl der therapierten Mammakarzinomfälle (=Zentralisierung)

- Diesbezüglich besondere Bedeutung der Gesamtzahl der Operationen pro Zentrum bzw. jeweilige Zahl der Operationen pro Operateur (=Spezialisierung)

- Große Bedeutung der Interdisziplinärität

Diese Kernaussagen, vor 2003 noch als offene Fragen formuliert, führen eindeutig auf eine wesentliche Qualitätsverbesserung hin, wie im Folgenden dargestellt wird.

\section{Bedeutung der Zentrumsbildung}

Die konkrete Frage, ob eine Zentralisierung der Behandlung des Mammakarzinoms auf Krankenhäuser mit einer Mindestanzahl an Fällen pro Jahr das Überleben der Patientinnen signifikant verbessern kann, wurde unter anderem in der wegweisenden Studie von Roohan et al. [9] beantwortet. Sie evaluierten die 5-JahresÜberlebensrate von 47890 Patientinnen in einem Zeitraum von 1984-1989 in 266 Krankenhäusern New Yorks im Zusammenhang mit der Fallzahl pro Jahr und Krankenhaus.

\section{Bedeutung der Spezialisierung}

Es ist bereits seit längerem nachgewiesen, dass das Gesamtüberleben mit der Spezialisierung der Ärzte steigt, die an der Diagnostik und Therapie des Mammakarzinoms beteiligt sind [6].

\section{Rolle der Spezialisierung des Operateurs}

Obwohl der Zusammenhang Spezialisierung und besseres Outcome häufiger nicht der chirurgischen Erfahrung des Operateurs zugeschrieben wird, sondern eher der optimierten, d.h. leitlinienkonformen und individualisierten adjuvanten Therapie, fanden Sainsbury et al. [10] genau diesen signifikanten Zusammenhang zwischen Operationszahl pro Operateur und Jahr und Verbesserung der 5-Jahres-Überlebensrate.

\section{Bedeutung der Interdisziplinarität}

In allen Studien wurde betont, dass die Interdisziplinarität für den Überlebensvorteil eine wesentliche Rolle spielt. So wurde bereits in der bekannten Metaanalyse von Richards et al. [8] festgestellt, dass die 5-Jahres-Überlebensrate besser ist, wenn in einem interdisziplinären Zentrum behandelt wurde und der Operateur mehr als 30-50 neue Fälle von Brustkrebs pro Jahr therapiert. 


\section{Benchmarking als „Proof of Principle“}

$\nabla$

Das Benchmarking stellt einen kontinuierlichen Prozess zur systematischen Verbesserung der Versorgungsqualität dar. Voraussetzung für das Benchmarking ist jedoch der Aufbau einer Infrastruktur, um die Qualitätsindikatoren zusammen zu führen und einen Vergleich durchführen zu können. Qualitätsindikatoren sind klinische Messgrößen, die in einem definierten Beobachtungszeitraum die Qualität eines Brustzentrums wider spiegeln sollen.

Langfristig sind allerdings als Indikatoren bei der Behandlung des Mammakarzinoms vor allem die Morbidität und Mortalität von zentralem Interesse, d.h. die Ergebnisqualität. Lokalrezidive bzw. Metastasierungen werden jedoch häufig erst in 5- bis 10Jahres-Zeiträumen evident.

Zentrumsbildungen und Zertifizierungswesen sind jedoch ihrer Effekte bisher kaum fundamental wissenschaftlich untersucht worden. Um dies für die interdisziplinären Brustzentren festzustellen, wurde in Deutschland erstmals eine prospektive multizentrische Studie im Zeitraum 2003-2008 durchgeführt [2].

Im Ergebnis erhöhte sich die primäre Anzahl der teilnehmenden BZ zwischen 2003 und 2008 von 59 auf 229, die der postoperativ gesicherten primären Brustkrebserkrankungen von 5994 auf 36914 (74\% der jährlichen Neuerkrankungen in Deutschland). Bis 2008 wurden (ausgehend von 9) 15 QIs als Surrogatindikatoren für die langfristige Ergebnisqualität entwickelt. Von 2003 bis 2008 konnten starke Steigerungen für diese QIs beobachtet werden: für die präoperative Diagnosesicherung von 58 auf $93 \%$, antihormonelle Therapie bei positiven Hormonrezeptoren von 27 auf $96 \%$, adjuvante und neoadjuvante Systemtherapie (ohne Altersbegrenzung) von 32 auf $71 \%$ sowie Bestrahlungen nach brusterhaltender Therapie von 20 auf $80 \%$ bzw. Mastektomie von 8 auf 65\%. A Abb. 1 zeigt beispielhaft die Entwicklung des relativen Erfüllungsgrades der Qualitätsindikatoren.

Die Ergebnisse sind bemerkenswert: Die Akzeptanz des Benchmarking-Konzeptes wird belegt durch die Steigerung der Behandlungsfälle in den teilnehmenden DKG/DGS-zertifizierten Brustzentren in Deutschland. Erstmalig wurden so valide Aussagen zur Versorgungsrealität für Deutschland möglich; die Erfassung von Longitudinalverläufen scheint gewährleistet!

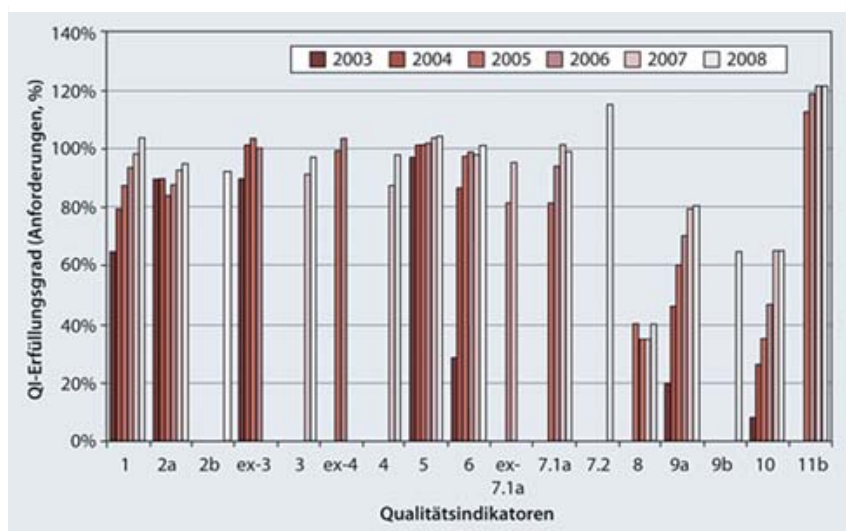

Abb. 1 Relativer Erfüllungsgrad der Qualitätsindikatoren (QIs) im Zeitraum 2003-2008 als Prozentsatz der jeweiligen Vorgabe für das dritte Jahr der Zertifizierung gemäß den Fachlichen Anforderungen an Brustzentren (FAB).
Zertifizierung von Brustzentren als „Proof of Concept" Von speziellem Interesse ist außerdem, inwieweit sich der Prototyp „Brustzentrum“ auf andere onkologische Zentrumsbildungen übertragen lässt.

Die erste „Pilotzertifizierung“ eines Brustzentrums nach ISO 9001 und den DKG / DGS Anforderungen (FAB) konnte Dezember 2002 erreicht werden.

Ein „Proof of concept“ wurde durch die ersten Rezertifizierungen erreicht. Ende 2005 konnten die ersten beiden DKG/DGS-zertifizierten Brustzentren nach der ersten Drei-Jahres-Zertifizierung erfolgreich rezertifiziert werden. Mitte 2008 hatten 79 von 80 Brustzentren die Rezertifizierung erfolgreich absolviert. Ein Zentrum hatte 2007 die Rezertifizierungs-Anforderderungen nicht erfüllen können und wurde deshalb vom weiteren DKG/ DGS Zertifizierungsprogramm ausgeschlossen.

In den Jahren 2004 bis 2009 (Stand 31.10.09) stieg die Anzahl der zertifizierten Zentren von 57 auf 195 an, davon waren 147 Zentren Einzelstandorte. Die Zahl der Zentren mit 2Standorten blieb konstant bei $44 Z$ Zentren, wobei sich allerdings der Anteil prozentual verringerte zugunsten der Einzelstandortzentren.

Innerhalb der 4,75Jahre zwischen Januar 2005 und Oktober 2009 stieg die Anzahl der Primärerkrankungen eines Brustzentrums innerhalb des ersten Jahres nach Zertifizierung im Schnitt um 6\% von 196 auf 239, während die Gesamtanzahl der behandelten primären Brustkrebserkrankungen an den Brustzentren, die nach dem dualen System zertifiziert waren um das 4-Fache von 11152 auf 46664 Fälle zunahm. Somit waren ca. $82 \%$ der in Deutschland geschätzten jährlichen 57000 Brustkrebsneuerkrankungen in einem zertifizierten Brustzentrum diagnostiziert und behandelt worden.

Das Ergebnis dieser aktuellen deskriptiven und bisher einzigartigen Studie von Brucker et al. [1] zeigt, dass die freiwillige Zertifizierung von Brustzentren in Deutschland gut akzeptiert ist. Insgesamt ist mittlerweile das DKG/DGS-Zertifizierungsprozedere für multidisziplinäre Brustzentren in Deutschland als Modell für die Qualitätssicherung und Zentralisierung von Zentren aus anderen Krebsbereichen anerkannt.

\section{Onkologische Qualitätsoptimierung in der Mamma- karzinomversorgung an zertifizierten deutschen Brustzentren unter besonderer Berücksichtigung eines Querschnittsfaches, der Radioonkologie $\nabla$}

Ziel der Untersuchung war es, anhand der aktuellen Daten von 2009 zu zeigen, dass entsprechende Analysen auch für einzelne Querschnittsfächer wie die Radioonkologie möglich sind [3]. Brustzentren wurden zur Teilnahme am Benchmarking eingeladen. Es wurden zunächst neun Qualitätsindikatoren (QI) aus leitlinienbasierten Qualitätszielen abgeleitet, die jährlich überprüft und durch Modifikation, Neueinführung oder Aufgabe von QI weiterentwickelt wurden. Die zeitlichen Veränderungen insbesondere der radioonkologisch relevanten QIs wurden deskriptiv ausgewertet. Im Untersuchungszeitraum 2003-2009 nahmen die Zahlen der am Benchmarking teilnehmenden Einrichtungen und der erfassten postoperativ gesicherten primären Brustkrebserkrankungen markant zu. Bis 2009 wurden, von neun Prozessindikatoren ausgehend, $15 \mathrm{QI}$ als Surrogatindikatoren für die langfristige Ergebnisqualität entwickelt. Bei zwei der sieben radioonkologisch relevanten QI (Bestrahlungen nach brusterhaltender Therapie bzw. Mastektomie) ergaben sich von 2003-2009 erhebliche Steigerungen (von 20 auf 85 bzw. 8 auf 70\%). Drei weitere, 
bereits initial hohe QI erreichten praktisch die Vorgabewerte. Mit den aktuellen Daten bestätigt sich der Proof-of-Concept für das etablierte Benchmarkingsystem, welches den Vergleich teilnehmender Einrichtungen sowie die Beobachtung zeitlicher Veränderungen in der Brustkrebsversorgungsqualität des gesamten Netzwerks erlaubt. Deutliche QI-Zuwächse weisen für 20032009 insgesamt auf Verbesserungen in der Brustkrebsversorgung in Deutschland hin. Erstmals sind Verbesserungen der Versorgungsqualität aber auch als Longitudinalverläufe für einzelne Brustzentren darstellbar. Anhand von Teilspektren relevanter QI lassen sich zudem Fortschritte, aber auch der weitere Verbesserungsbedarf für einzelne Querschnittsfächer nachweisen.

\section{Zusammenfassende Wertung \\ $\nabla$}

Übergeordnetes Ziel aller an der Behandlung des Mammakarzinoms Beteiligten bleibt die Senkung der Mortalitätsrate, die Verbesserung der Lebensqualität sowie die Verlängerung des Überlebens. Voraussetzung dafür ist die Qualitätssicherung auf der Basis eines multidisziplinären, spezialisierten Managements in einem qualitätsgesicherten, zertifizierten Schwerpunkt mit einem Qualitätsmanagementsystem (QMS), das regelmäßig durch unabhängige Audits überprüft wird. Diese Brustkrebsschwerpunkte müssen nun noch stärker in ein überregionales Verbundsystem eingebunden werden, indem die Versorgung nach (europäischen) Leitlinien stattfindet, Studien durchgeführt werden und deren Daten innerhalb des Verbunds einheitlich dokumentiert werden sowie ein gemeinsames Benchmarking basierend auf einheitlich definierten Qualitätsindikatoren durchgeführt wird. Der Erfolg dieser Qualitätssicherungsmaßnahmen wird dann mit Hilfe einer gut strukturierten Dokumentation anhand der Ergebnisqualität und des Erfüllungsgrades für die einzelnen Qualitätsindikatoren gemessen.

Hinsichtlich der Wertigkeit von Zentrum- oder Schwerpunktbildung bzw. Zertifizierung lässt sich folgern, dass es zwar einen positiven Zusammenhang zwischen Fallzahl pro Jahr pro Krankenhaus bzw. Fallzahl pro Jahr pro Operateur und der Verbesserung der Überlebensraten gibt, diese Zahlen aber strengen statistischen Kriterien nicht immer unbedingt standhalten. Fest steht allerdings, dass Interdisziplinarität und Qualitätssicherung zur Verbesserung der onkologischen Ergebnisse entscheidend beitragen. Durch die Einführung einer Qualitätssicherung erfolgt darüber hinaus auch ein Bewusstmachen der Prozessabläufe und damit die Verbesserung von interdisziplinärer Kooperation und somit eine Verbesserung der Versorgung der Patientin.

Über die Flächendeckung hinaus muss die Implementierung von Leitzentren gefördert werden, die den gemeinsamen Datentransfer zum Benchmarking bzw. die Benchmarkinganalyse mitbegleiten. Solche „center of excellence“ sind vor allen Dingen für den Wissenstransfer, die Studienrekrutierung, die wissenschaftliche Auswertung und die Implementierung der Leitlinien auf neuestem Stand unabdingbare Voraussetzungen. Auf dieser Basis kann eine Harmonisierung der Qualitätsanforderungen zwischen den wissenschaftlichen Fachgesellschaften und der nationalen und europäischen Ebene angestrebt und auch umgesetzt werden. Neben der Notwendigkeit der Vereinfachung der dargestellten Benchmarkingverfahren, der Ermöglichung kostengünstiger zentraler Verfahren bzw. der Endbürokratisierung der Qualitätssicherung und Zertifizierung sollte nach wie vor das gesetzliche Mammografiescreening unter Miteinbeziehung des Netzwerkes der Niedergelassenen Fachärzte direkt mit den zertifizierten
Brustzentren verbunden werden. Ebenso ist vor dem Hintergrund des enormen und darüber hinaus nicht vergüteten Aufwandes der Dokumentation, des Benchmarkings- und der Qualitätssicherung undenkbar, dass verschiedene Sicherungssysteme nicht harmonisiert nebeneinander existieren! Dabei muss betont werden, dass eine Vereinheitlichung nationaler mit internationalen logischen Zertifizierungsverfahren ebenso noch nicht umgesetzt sind.

Das Mammakarzinom ist aufgrund seiner hohen Inzidenz und der Erfordernis einer interdisziplinärer Versorgung besonders geeignet für eine Überprüfung, ob das diesbezüglich angewandte Instrumentarium eines nationalen Benchmarking als Prototyp für onkologische Zentrumsbildungen fungieren kann. Zukünftig muss das Benchmarking aber nicht nur auf der Basis des XMLDatensatzes weiter standardisiert werden, sondern auch die konkurrierenden Systeme in Deutschland DOC vs. BQS müssen gegenüberstellend evaluiert werden und ggf. harmonisiert werden. Problematisch ist auch, dass mindestens 1/3 aller Brustzentren eigene Benchmarking-Systeme nutzen.

\section{Fazit}

$\nabla$

Aktuelle sowohl epidemiologische als auch gesundheitsökonomische Evaluierungen verdeutlichen die Relevanz von Zentrumsbildungen für die Qualität der Versorgung von Mammakarzinompatientinnen. Konkret deuten Verbesserungen im Bereich von Struktur- und Prozessqualität im Sinne von Surrogat-Parameter auf eine Verbesserung bei der Ergebnisqualität hin. Auch wenn das Ziel der Etablierung eines deutschlandweiten Netzwerkes von zertifizierten Brustzentren als weitgehend erreicht bezeichnet werden kann, und die Akzeptanz des Benchmarking-Konzeptes in den teilnehmenden DKG/DGS-zertifizierten Brustzentren in Deutschland nachgewiesen hoch ist, so besteht im Bereich des Versorgungsnetzwerks, vom Mammografie-Screening über interventionelle Diagnostik bis zur Nachsorge, nach wie vor Optimierungsbedarf. Spezialisierung, leitliniengerechtes Vorgehen und Zertifizierung bzw. Rezertifizierung sind weiterhin essentiell um die bisher erreichte verbesserte Versorgungsqualität zu optimieren und die flächendeckende Versorgung zu stabilisieren.

\section{Literatur}

1 Brucker SY, Bamberg M, Jonat W et al. Certification of breast centres in Germany: proof of concept for a prototypical example of quality assurance in multidisciplinary cancer care. BMC Cancer 2009; 9: 228

2 Brucker SY, Schumacher C, Sohn C and the Steering Committee. Benchmarking the quality of breast cancer care in a nationwide voluntary system: the first five-year results (2003-2007) from Germany as a proof of concept. BMC Cancer 2008; 8: 358

3 Brucker SY, Wallwiener M, Kreienberg R et al. Optimizing the Quality of Breast Cancer Care at Certified German Breast Centers. A Benchmarking Analysis for 2003-2009 with a Particular Focus on the Interdisciplinary Specialty of Radiation Oncology. Strahlenther Onkol 2011; 187: 89 - 99

4 Bundesministerium für Gesundheit. Maßnahmen des Bundesministeriums für Gesundheit zur Umsetzung der nationalen Gesundheitsziele. Berlin: 2007

5 Ferlay J, Autier P, Boniol $M$ et al. Estimates of the cancer incidence and mortality in Europe in 2006. Ann Oncol 2007; 18: 581 - 592

6 Giersiepen K, Heitmann C, Janhsen K et al. Brustkrebs. Gesundheitsberichterstattung des Bundes (GBE). Heft 25; Berlin: Robert KochInstitut in Zusammenarbeit mit dem Statistischen Bundesamt; 2005

7 Krebs in Deutschland 2003-2004. Häufigkeiten und Trends. 6. überarbeitete, aktualisierte Aufl. Saarbrücken: Gesellschaft der epidemiologischen Krebsregister in Deutschland e.V. und das Robert KochInstitut; 2008 
8 Richards $M$, Sainsbury $R$, Kerr D. Inequalities in breast cancer care and outcome. Br J Cancer 1997; 76: 634-638

9 Roohan PJ, Bickell NA, Baptiste MS et al. Hospital volume differences and five-year survival from breast cancer. Am J Public Health 1998; 88: $454-457$

10 Sainsbury R, Howard B, Rider L et al. Influence of clinician workload and pattern of treatment on survival from breast cancer. Lancet 1995; 345 : $1265-1270$

11 Schön D, Bertz J, Görsch B et al. Die Dachdokumentation Krebs. Eine Surveillance-Einrichtung der Krebsregistrierung in Deutschland. Bundesgesundheitsblatt Gesundheitsforschung Gesundheitsschutz 2004; 47: $429-436$
Bibliografie

DOI http://dx.doi.org/ 10.1055/s-0031-1271511

Senologie 2011; 8: 92-95

(c) Georg Thieme Verlag KG Stuttgart · New York · ISSN 1611-6453

\section{Korrespondenzadresse}

\section{Prof. Dr. med. Diethelm Wallwiener}

Ärztlicher Direktor der Universitäts-Frauenklinik

Calwerstr. 7

72076 Tübingen

diethelm.wallwiener@med.uni-tuebingen.de

Gekürzter Nachdruck des Beitrags: D. Wallwiener, M. Bamberg, W. Jonat, R. Kreienberg and S. Brucker. Zertifizierte multidisziplinäre Brustzentren. Ein Implementierungsprojekt der Deutschen Krebsgesellschaft und der Deutschen Gesellschaft für Senologie in Partnerschaft mit der Deutschen Gesellschaft für Gynäkologie und Geburtshilfe. Der Gynäkologe, 2010, Volume 43, Number 3, Pages 205-215.

Mit freundlicher Genehmigung von Springer Science and Business Media. 